\title{
Long noncoding RNA LINCOI 296 is associated with poor prognosis in prostate cancer and promotes cancer-cell proliferation and metastasis
}

This article was published in the following Dove Press journal:

OncoTargets and Therapy

27 March 2017

Number of times this article has been viewed

\section{Jie $\mathrm{Wu}^{*}$ \\ Gong Cheng* \\ Cheng Zhang* \\ Yuxiao Zheng \\ Haoxiang Xu \\ Haiwei Yang \\ Lixin Hua}

Department of Urology, First Affiliated Hospital of Nanjing Medical University, Nanjing, China

*These authors contributed equally to this work
Correspondence: Lixin Hua;

Haiwei Yang

Department of Urology, First Affiliated Hospital of Nanjing Medical University, 300 Guangzhou Road, Nanjing, Jiangsu 210029, China

Tel +86 I30 7256 4303;

$+8613951762701$

Email lixinhua@njmu.edu.cn;

haiweiyang@njmu.edu.cn
Background/purpose: Long noncoding RNAs (lncRNAs) have emerged as important regulators and biomarkers of tumor development and progression. This study investigated the clinical significance, biological functions, and underlying mechanisms of long intergenic nonprotein-coding RNA 1296 (LINC01296) in prostate cancer.

Materials and methods: LINC01296 expression in prostate cancer tissues and cell lines was detected by quantitative real-time polymerase chain reaction (qRT-PCR). The correlation between LINC01296 expression and clinicopathologic characteristics of prostate cancer was analyzed using Kaplan-Meier analysis and the Cox proportional-hazard model. Small interfering RNA was used to suppress LINC01296, and knockdown efficiency was examined by qRT-PCR. Cell Counting Kit 8 assay, colony-formation assay, migration and invasion assays, and Western blot assay were used to explore the role of LINC01296 in tumor progression further.

Results: LINC01296-expression level was higher in prostate cancer tissues and prostate cancer cells than in adjacent nontumor tissues and immortalized normal prostate stromal WPMY1 cells. LINC01296 expression was correlated with preoperative prostate specific antigen $(P=0.002)$, lymph-node metastasis $(P=0.035)$, Gleason score $(P<0.001)$, and tumor stage $(P=0.036)$. Patients with higher LINC01296 expression displayed advanced clinical features and shorter biochemical recurrence-free survival time than those with lower LINC01296 expression. Multivariate analysis showed that LINC01296 expression was an independent predictor of biochemical recurrence-free survival in prostate cancer. Additionally, LINC01296 knockdown inhibited prostate cancer-cell proliferation, migration, and invasion, demonstrated in an in vitro study involving regulation of PI3K-Akt-mTOR signaling and epithelial-mesenchymal transition.

Conclusion: The results demonstrated that LINC01296 is a novel molecule involved in prostate cancer development and progression, and thus is a potential biomarker of prognosis.

Keywords: long noncoding RNA, prostate cancer, prognosis, proliferation, migration, invasion

\section{Introduction}

Prostate cancer is one of the most common malignant tumors in males worldwide, particularly in developed Western countries. ${ }^{1,2}$ The latest data indicate that the incidence and mortality of prostate cancer are estimated to reach up to 180,890 new cases and 85,920 new deaths by the year 2016 in the US. ${ }^{3}$ In addition, the incidence and mortality of prostate cancer among Asian men has increased rapidly during the past decade. ${ }^{4}$ In recent years, the incidence of prostate cancer in China has also increased rapidly, and this phenomenon is associated with lifestyle changes; moreover, increased detection of prostate cancer is attributed to prostate-specific antigen screening and improved 
biopsy techniques. ${ }^{5}$ Early-stage prostate cancer patients often show good prognosis after comprehensive treatment. Nevertheless, recurrence, metastasis, and development of castration-resistant prostate cancer remain the leading causes of mortality. The detailed molecular mechanisms underlying prostate cancer carcinogenesis remain to be fully elucidated. Therefore, studies on prostate cancer remain extremely important.

Recent evidence has indicated that noncoding RNAs (ncRNAs) play a key role in cancer. Based on their sizes, ncRNAs can be classified into two major groups: small ncRNAs (eg, microRNAs) and long ncRNAs (lncRNAs). lncRNAs are a class of transcripts that are longer than 200 nucleotides and do not contain an open reading frame. ${ }^{6,7}$ IncRNAs have been proved to be involved in the development and progression of prostate cancer; however, research on the involvement of IncRNA in prostate cancer is still in its preliminary stage.

Based on the relationship between IncRNA and prostate cancer, IncRNA can be roughly divided into three categories: carcinogenicity lncRNA, tumor-suppressor lncRNA, and lncRNA with unknown functions. ${ }^{8} P C A 3$, also known as carcinogenicity lncRNA, is the first lncRNA to be identified in prostate cancer, and has been used in different applications, including diagnosis, treatment, and prediction of prostate cancer. ${ }^{910}$ Additionally, MALAT1, PCAT1, and SCHLAP1 demonstrate oncogenic functions in prostate cancer. ${ }^{11-13}$ PCGEM1 is an androgen-induced prostate-specific lncRNA that is highly expressed in early stages of prostate cancer; ${ }^{14}$ PCGEM1 was also detected in African-American prostate cancer patients or in prostate cancer patients with a family history of prostate cancer. ${ }^{15}$ PCGEM1 is associated with the targeted androgen receptor, which contributes to the development of castration-resistant prostate cancer. ${ }^{16}$ In addition, $M E G 3$ is a tumor suppressor. MEG3 can promote apoptosis and inhibit proliferation by stabilizing p 53 expression and by selectively activating the expression of the p53 target gene. ${ }^{17,18}$ IncRNA H19 plays a tumor-suppressive role in metastatic prostate cancer by repressing the effects of TGF $\beta_{1} \cdot{ }^{19}$ However, newer lncRNAs are being investigated through high-throughput technologies. Moreover, the functional role and underlying molecular mechanisms of the lncRNAs in the development and progression of prostate cancer remain unclear.

With the rapid development of new-generation sequencing, RNA-sequencing technology has been widely used to analyze the transcriptomes of various organisms; this technology is useful in the discovery of new therapeutic targets and prognostic indicators. ${ }^{20,21}$ Our previous microarray data have revealed the novel intergenic IncRNA LINC01296 to be significantly upregulated $(P<0.05$, fold-change $>2)$. LINC01296 is located at chromosome 14q11.2.

In this study, we analyzed the expression of the novel IncRNA LINC01296 (ENSG00000244306) in prostate cancer tissue samples and investigated its potential biomedical functions. Our results demonstrated that LINC01296 is a potential new molecular biomarker or a new basis for prostate cancer treatment.

\section{Materials and methods}

\section{Ethics statement}

Written informed consent was obtained from all participants involved in this study. This research was approved by the institutional review board of the First Affiliated Hospital of Nanjing Medical University (Nanjing, China).

\section{Patient samples and microarray analysis}

Prostate cancer tissues and paired adjacent normal tissues were obtained from 73 patients who had undergone surgical resection of prostate cancer from February 2009 to April 2014. After resection, all tissue samples were immediately frozen and stored in liquid nitrogen until further analysis. The diagnosis of prostate cancer was confirmed by histopathology. Three sample pairs were used for microarray analysis, and other pairs were used for additional studies. A total of 8,277 IncRNA probes were used in microarray assays. Differentially expressed lncRNAs were identified by comparing the expression levels in tumor samples and in adjacent normal samples.

\section{Cell lines and culture conditions}

Human prostate cancer cell lines (22Rv1 and LNCaP) and a normal myofibroblast stromal cell line (WPMY1) were purchased from the Institute of Biochemistry and Cell Biology of the Chinese Academy of Sciences (Shanghai, China). These cells were cultured in Roswell Park Memorial Institute 1640 medium or Dulbecco's Modified Eagle's Medium (Thermo Fisher Scientific, Waltham, MA, USA) supplemented with $10 \%$ fetal bovine serum, $100 \mathrm{mg} / \mathrm{mL}$ streptomycin, and $100 \mathrm{U} / \mathrm{mL}$ penicillin (Thermo Fisher Scientific) in a humidified atmosphere at $37^{\circ} \mathrm{C}$ with $5 \% \mathrm{CO}_{2}$.

\section{RNA isolation and quantitative real-time polymerase chain reaction}

Total RNA was extracted from tissues or cultured cell lines using Trizol reagent (Thermo Fisher Scientific) according 
to the manufacturer's protocols. For reverse-transcription polymerase chain reaction (PCR), $1 \mu \mathrm{g}$ of total RNA was reverse-transcribed to complementary DNA in a final volume of $10 \mu \mathrm{L}$ by using random primers $(10 \mu \mathrm{M})$ and a reverse-transcription kit (Takara, Kyoto, Japan). Reverse transcription was performed at $37^{\circ} \mathrm{C}$ for 15 minutes and then at $85^{\circ} \mathrm{C}$ for 5 seconds and $4^{\circ} \mathrm{C}$ for 5 minutes according to the manufacturer's instructions. Quantitative real-time PCR (qRT-PCR) was performed on an ABI StepOnePlus (Thermo Fisher Scientific) using a standard protocol from Power SYBR Green (Takara) according to the manufacturer's instructions; the reaction was performed in $10 \mu \mathrm{L}$ reaction volume containing $5 \mu \mathrm{L}$ of SYBR Premix $(2 \times)$, $0.4 \mu \mathrm{L}$ of PCR forward primer $(10 \mu \mathrm{M}), 0.4 \mu \mathrm{L}$ of PCR reverse primer $(10 \mu \mathrm{M}), 0.2 \mu \mathrm{L}$ of ROX Reference Dye II $(50 \times), 3 \mu \mathrm{L}$ of double-distilled water, and $1 \mu \mathrm{L}$ of complementary DNA of the sample. qRT-PCR was performed under the following conditions: $95^{\circ} \mathrm{C}$ for 10 minutes, $95^{\circ} \mathrm{C}$ for 30 seconds, $95^{\circ} \mathrm{C}$ for 5 seconds, $61^{\circ} \mathrm{C}$ for 30 seconds in cycles for a total of 50 cycles, and a final extension step at $72^{\circ} \mathrm{C}$ for 5 minutes. $\Delta \mathrm{Ct}$ values were normalized to $\beta$-actin. The relative quantitative value was obtained using the $2^{-\Delta \Delta \mathrm{Ct}}$ method. Relative quantitative values greater than 2 were defined as high expression, and less than 0.5 as low expression. Each experiment was performed in triplicate and repeated three times. The primers were: LINC01296, forward 5'-AAGTGGCACCAGCCTCACT-3', reverse 5'-CGGCCAAGT TCTTTACCATC-3'; $\beta$-actin, forward 5'-ACTGGAACGGTGAAGGTGA C-3', reverse 5'-AGAGAAGTGGGGTGGCTTTT-3'.

\section{Small interfering RNA transfection}

Small interfering RNA (siRNA) that targets LINC01296 RNA and unspecific control was purchased from GenePharma (Shanghai, China). The target sequences for siRNA-LINC01296 were: siRNA-LINC01296-1 (S1), 5'-GGCUGGAGAAUAUUUCCUATTTT-3'; SiRNA-LINC01296-2 (S2), 5'-CUGAAACAUAUU CCGUGGUTT-3'; and siRNA-LINC01296-3 (S3), 5'-GCAGCUGGGAUUUGUAAGUTT-3'. The experiments showed that $\mathrm{S} 1$ and $\mathrm{S} 2$ demonstrated the highest inhibition efficiency and thus were used in the subsequent experiments. Synthetic sequence-unspecific siRNA was used as negative control (NC) siRNA. Prior to transfection with siRNA-LINC01296 and NC siRNA using Lipofectamine 2000 (Thermo Fisher Scientific) according to the manufacturer's instructions, the cells were cultured in six-well plates containing complete growth media until cell density reached $60 \%$. The cells were harvested after 48 hours for qRT-PCR and Western blot analyses.

\section{Cell-proliferation assays}

A CCK-8 assay (Dojindo, Kumamoto, Japan) was used to analyze the proliferation of the siRNA-transfected cell lines. After siRNA transfection for 48 hours, 2,000 cells per well were incubated in 96-well plates, each well containing $200 \mu \mathrm{L}$ of complete culture medium. After 6-8 hours of culture and 24, 48, 72, and 96 hours, the cells were treated with $10 \mu \mathrm{L}$ of CCK-8, which was added into the complete culture medium. The cells were incubated at $37^{\circ} \mathrm{C}$ for 4 hours. Absorbance was measured at $450 \mathrm{~nm}$. All experiments were performed in triplicate.

\section{Colony-formation assay}

For the colony-formation assay, cells were trypsinized into single-cell suspensions after transfection. A total of 1,000 cells were plated into each median plate and maintained in complete culture medium, which was replaced every 3-5 days. After 14 days, colonies were fixed with $4 \%$ paraformaldehyde and stained with $0.1 \%$ crystal violet (Beyotime, Haimen, China) for 20 minutes. Finally, visible colonies ( $>50$ cells/colony) were manually counted after being washed twice with double-distilled $\mathrm{H}_{2} \mathrm{O}$. Triplicate plates were measured in each treatment group.

\section{Cell-migration and -invasion assays}

Cell-invasion and -migration assays were performed in a transwell chamber coated with or without Matrigel (BD Biosciences, San Jose, CA, USA) 48 hours after siRNA transfection. Cells $\left(2.5 \times 10^{4}\right)$ were suspended in $200 \mu \mathrm{L}$ of serum-free medium and seeded in the upper chamber of a transwell. Culture medium $(500 \mu \mathrm{L})$ containing $10 \%$ fetal bovine serum was placed into the bottom chamber. After incubation for 72 hours in a humidified atmosphere of $5 \% \mathrm{CO}_{2}$ at $37^{\circ} \mathrm{C}$, the cells on the upper surface of the transwell chambers were removed using cotton swabs. Cells on the bottom surface of the transwell chambers were fixed with polyoxymethylene and stained with $0.1 \%$ crystal violet for 20 minutes. The invaded cells were counted in five randomly selected fields under microscopy, and the average value was calculated. Each experiment was conducted in triplicate. Matrigel invasion assays were performed with the same methods.

\section{Protein extraction and Western blot assay}

Transfected cells were washed twice with cold phosphatebuffered saline and lysed using the mammalian protein-cleavage 
reagent radioimmunoprecipitation assay (Beyotime) supplemented with protease inhibitors (Hoffman-La Roche, Basel, Switzerland) and phenylmethylsulfonyl fluoride (Hoffman-La Roche) at $4^{\circ} \mathrm{C}$ for 30 minutes. Cellular debris were subsequently removed by centrifugation at $12,000 \mathrm{rpm}$ for 10 minutes. The supernatants were mixed with quartervolume $5 \times$ sodium dodecyl sulfate sample buffer and boiled for 10 minutes. Protein samples were separated by $8 \%-10 \%$ sodium dodecyl sulfate polyacrylamide-gel electrophoresis and then transferred onto polyvinylidene difluoride (PVDF) membranes (Bio-Rad Laboratories, Hercules, CA, USA). The PVDF membranes were soaked in 5\% w/v skim milk at room temperature for 2 hours and then incubated overnight with primary antibodies at $4^{\circ} \mathrm{C}$. The PVDF membranes were subsequently incubated with horseradish peroxidase-conjugated goat antirabbit secondary antibodies (Cell Signaling Technology, Danvers, MA, USA) for 1 hour at room temperature. An enhanced-chemiluminescence chromogenic substrate was used to visualize the bands, and the intensity of the bands was quantified by Image Lab software (Quantity One;
Bio-Rad). GAPDH antibody (Cell Signaling Technology) was used as control. Rabbit monoclonal antibodies of PI3K, Akt, mTOR, vimentin, E-catenin, N-cadherin, and MMP9 were purchased from Cell Signaling Technology.

\section{Statistical analysis}

All results are expressed as mean \pm standard deviation, and statistical analyses were performed using SPSS version 19.0 (IBM, Armonk, NY, USA). Student's $t$-test and $\chi^{2}$ tests were performed, and $P<0.05$ indicated statistical significance. The Kaplan-Meier method was applied for survival analysis, while related data were analyzed using Cox proportionalhazard regression model.

\section{Results}

\section{LINCOI 296 expression in prostate cancer tissues and cell lines}

lncRNA-expression profiles of three pairs of prostate cancer samples were screened through microarray data analysis (Figure 1A). qRT-PCR was used to examine the expression of
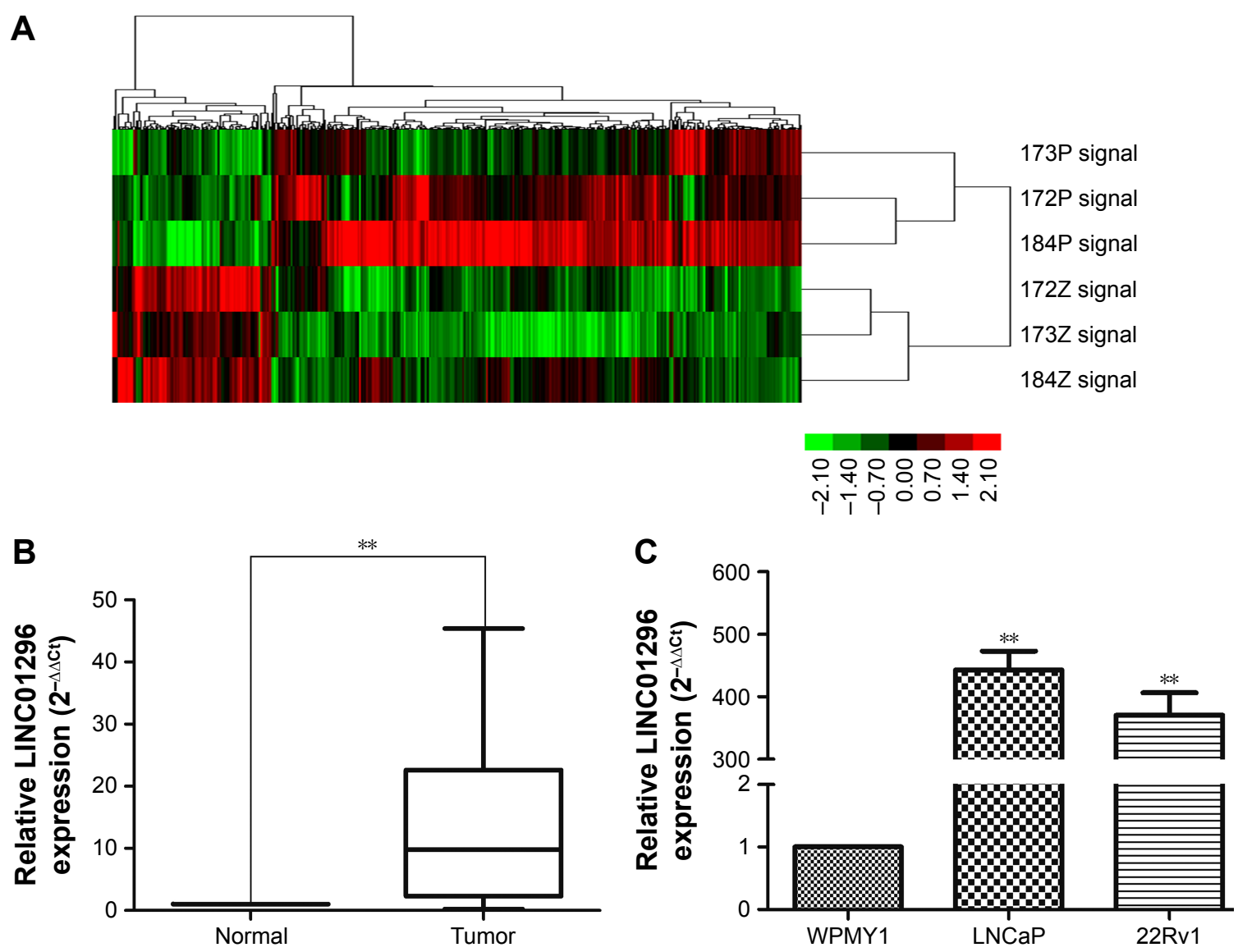

Figure I Relative LINCOI296 expression in prostate cancer.

Notes: (A) A microarray containing 8277 IncRNA probes was used to identify differentially expressed IncRNAs in three prostate cancer patients. (B) Relative expression of LINCOI 296 in prostate cancer tissues compared with paired adjacent normal tissues. (C) Relative expression of LINC0I296 in prostate cancer cell lines (22RvI, LNCaP) and normal prostate cell line (WPMYI). Each experiment was repeated three times. $* * P<0.01$.

Abbreviation: IncRNA, long noncoding RNA. 
LINC01296 in 70 pairs of prostate cancer tissues and adjacent normal tissues. The qRT-PCR result was consistent with the microarray data. Additionally, LINC01296 expression was significantly upregulated in prostate cancer tissues compared with paired adjacent normal tissues (Figure 1B). LINC01296 expression in two prostate cancer cell lines (22Rv1 and LNCaP) and in a normal human myofibroblast stromal cell line (WPMY1) was further detected by qRT-PCR. The result revealed that LINC01296 was overexpressed in 22Rv1 and LNCaP, but not in WPMY1 (Figure 1C).

\section{Correlations between LINCOI 296 expression and clinicopathologic features in prostate cancer patients}

Based on LINC01296 messenger RNA expression, patients were classified into two groups: a high LINC01296-expression group $(n=54)$ and a low LINC01296-expression group $(n=16)$. Clinicopathologic features of these groups were compared (Table 1). Statistical results demonstrated that the high LINC01296-expression group was significantly correlated with high preoperative prostate-specific antigen $(P=0.002)$, lymph-node metastasis $(P=0.035)$, Gleason score $(P<0.001)$, and tumor stage $(P=0.036)$ compared with the low LINC01296-expression group. LINC01296-expression level was not correlated with age $(P=0.569)$, prostate volume $(P=0.794)$, or multiple lesions $(P=0.171)$.

Table I The association between LINCOI296 expression and clinicopathological features of prostate cancer

\begin{tabular}{|c|c|c|c|c|}
\hline & \multirow[t]{2}{*}{ Total (\%) } & \multicolumn{2}{|c|}{ LINC0I 296 expression } & \multirow[t]{2}{*}{$P$-value } \\
\hline & & Low (\%) & High (\%) & \\
\hline \multicolumn{4}{|c|}{ Age (years) } & 0.569 \\
\hline$<65$ & $14(20)$ & $4(28.6)$ & $10(7 \mid .4)$ & \\
\hline$\geq 65$ & $56(80)$ & $12(21.4)$ & $44(78.6)$ & \\
\hline \multicolumn{4}{|c|}{ Prostate volume $\left(\mathrm{cm}^{3}\right)$} & 0.794 \\
\hline$<50$ & $37(52.9)$ & $8(21.6)$ & $29(78.4)$ & \\
\hline$\geq 50$ & $33(47.1)$ & $8(24.2)$ & $25(75.8)$ & \\
\hline \multicolumn{4}{|c|}{ Preoperative PSA (ng/mL) } & 0.002 \\
\hline$<10$ & $29(41.4)$ & $12(4 \mid .4)$ & $17(48.6)$ & \\
\hline$\geq 10$ & $4 \mathrm{I}(58.6)$ & $4(9.8)$ & $37(90.2)$ & \\
\hline \multicolumn{4}{|c|}{ Lymph-node metastasis } & 0.035 \\
\hline Presence & $38(54.3)$ & $5(\mid 3.2)$ & $33(86.8)$ & \\
\hline Absence & $32(45.7)$ & II (34.4) & $21(65.6)$ & \\
\hline \multicolumn{4}{|c|}{ Gleason score } & 0.001 \\
\hline$<7$ & $23(32.9)$ & $12(52.2)$ & II (47.8) & \\
\hline$\geq 7$ & $47(67.1)$ & $4(8.5)$ & $43(9 \mid .5)$ & \\
\hline \multicolumn{4}{|c|}{ Tumor stage } & 0.036 \\
\hline $\mathrm{T} 2$ & $28(40)$ & $10(35.7)$ & $18(64.3)$ & \\
\hline T3-T4 & $42(60)$ & $6(14.3)$ & $36(85.7)$ & \\
\hline \multicolumn{4}{|c|}{ Multiple lesions } & 0.171 \\
\hline Presence & $43(61.4)$ & $9(20.9)$ & $34(79.1)$ & \\
\hline Absence & $27(38.6)$ & 7 (25.9) & $20(74.1)$ & \\
\hline
\end{tabular}

Abbreviation: PSA, prostate specific antigen.

\section{Association between LINCOI 296 expression and prognosis of prostate cancer}

We further analyzed the correlation of LINC01296 expression with the prognosis of patients with prostate cancer after radical prostatectomy. Kaplan-Meier analysis results showed that prostate cancer patients with high LINC01296 expression displayed significantly shorter biochemical recurrence-free survival time than those with low LINC01296 expression ( $P=0.01$, Figure 2 ). As shown in Table 2, LINC01296-expression level and Gleason score were significantly correlated with biochemical recurrence-free survival rate of prostate cancer patients $(P<0.05)$. Univariate and multivariate analyses showed that the relative expression of LINC01296 and Gleason score were independent prognostic markers for biochemical recurrence-free survival of prostate cancer patients (Table 2). These data collectively indicated that high LINC01296 expression was an independent risk factor for prostate cancer patients.

\section{Knockdown of LINCOI296 inhibits prostate cancer-cell proliferation}

To verify the biological functions of LINC01296 in prostate cancer cell lines, we downregulated LINC01296 expression by transfecting siLINC01296 or an NC siRNA into 22Rv1 and LNCaP cells. qRT-PCR results showed that siLINC01296 demonstrated the highest inhibition efficiency,

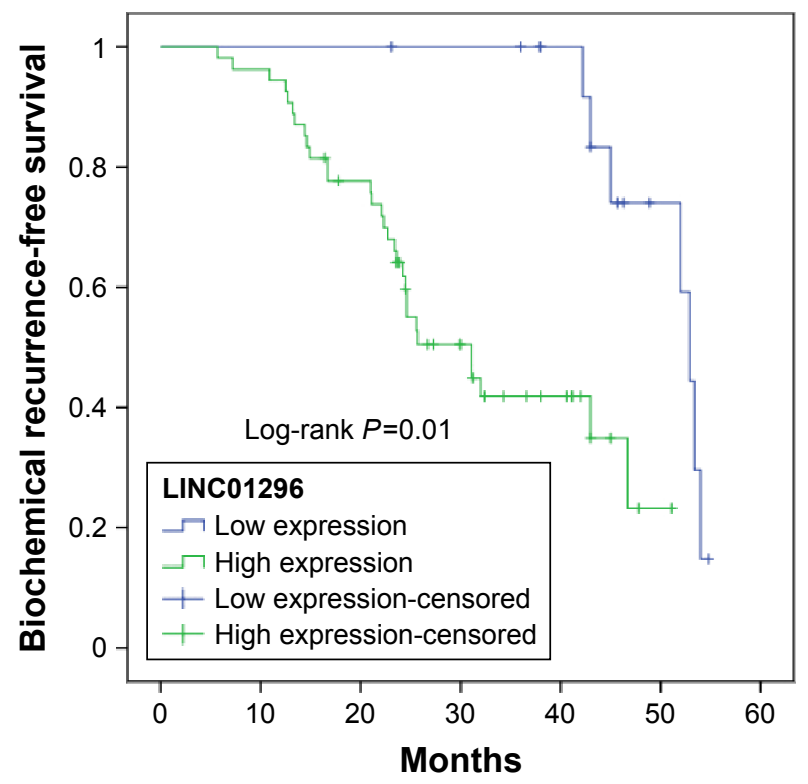

Figure 2 Kaplan-Meier survival curves of two groups of patients with prostate cancer based on LINCOI296-expression levels $(P=0.01)$.

Note: Patients with high levels of LINCOI296 expression showed reduced biochemical recurrence-free survival times compared with patients with low levels of LINC0I 296 expression. 
Table 2 Cox proportional-hazard model of biochemical recurrence-free survival in prostate cancer

\begin{tabular}{|c|c|c|c|c|c|c|}
\hline & \multicolumn{3}{|c|}{ Univariate analysis } & \multicolumn{3}{|c|}{ Multivariate analysis } \\
\hline & HR & $95 \% \mathrm{Cl}$ & $P$-value & HR & $95 \% \mathrm{Cl}$ & $P$-value \\
\hline Age (years) & & & 0.675 & & & \\
\hline$\geq 65$ vs $<65$ & 1.18 & $0.545-2.552$ & & & & \\
\hline Prostate volume $\left(\mathrm{cm}^{3}\right)$ & & & 0.78 & & & \\
\hline$\geq 50$ vs $<50$ & 0.911 & $0.476-1.746$ & & & & \\
\hline Preoperative PSA (ng/mL) & & & 0.09 & & & \\
\hline$\geq 10$ vs $<10$ & 1.879 & $0.906-3.898$ & & & & \\
\hline Lymph-node metastasis & & & 0.406 & & & \\
\hline Presence vs absence & 1.319 & $0.687-2.535$ & & & & \\
\hline Gleason score & & & 0.006 & & & 0.041 \\
\hline$\geq 7$ vs $<7$ & 3.429 & $1.426-8.247$ & & 2.965 & $1.047-8.397$ & \\
\hline Tumor stage & & & 0.491 & & & \\
\hline T3-T4 vs T2 & 1.272 & $0.642-2.519$ & & & & \\
\hline Multiple lesions & & & 0.514 & & & \\
\hline Presence vs absence & 0.799 & $0.407-1.569$ & & & & \\
\hline LINCOI 296 expression & & & 0.002 & & & 0.044 \\
\hline High vs low & 6.584 & $1.951-22.224$ & & 4.003 & $1.038-15.67$ & \\
\hline
\end{tabular}

Abbreviations: $\mathrm{HR}$, hazard ratio; $\mathrm{Cl}$, confidence interval; PSA, prostate specific antigen.

approximately $77 \%$ and $86 \%$ in $22 \mathrm{Rv} 1$ and LNCaP cell lines, respectively (Figure 3A, $P<0.05$ ). CCK-8 assays were used to detect the impact of LINC01296 knockdown on proliferation of prostate cancer cells. Results shown that knockdown of LINC01296 expression significantly decreased cell growth compared with NC siRNA transfection at 96 hours in 22Rv1 and LNCaP cell lines (Figure 3B, $P<0.05$ ).

A

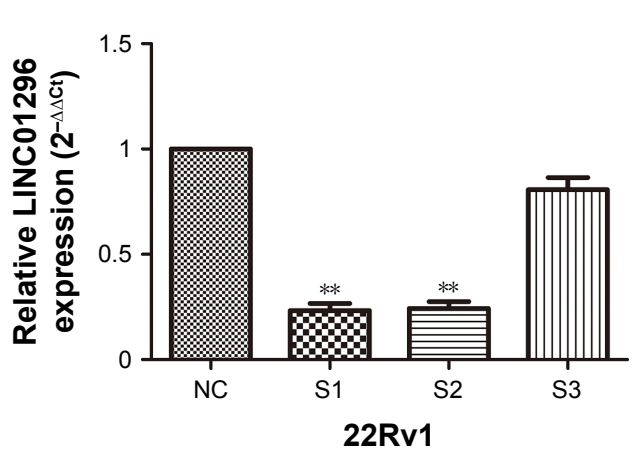

B

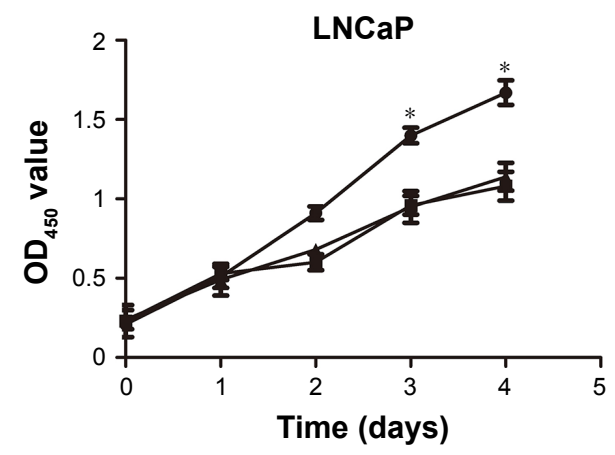

Cell colony-formation assays were used to investigate further the effect of LINC01296 on proliferation of 22Rv1 and LNCaP cells. The results showed that the colonyformation numbers of $22 \mathrm{Rv} 1$ and $\mathrm{LNCaP}$ cells transfected with siLINC01296 were obviously lower than those transfected with NC siRNA (Figure $3 \mathrm{C}$ and $\mathrm{D}, P<0.05$ ). All of the results were consistent with those of cell-proliferation assays.
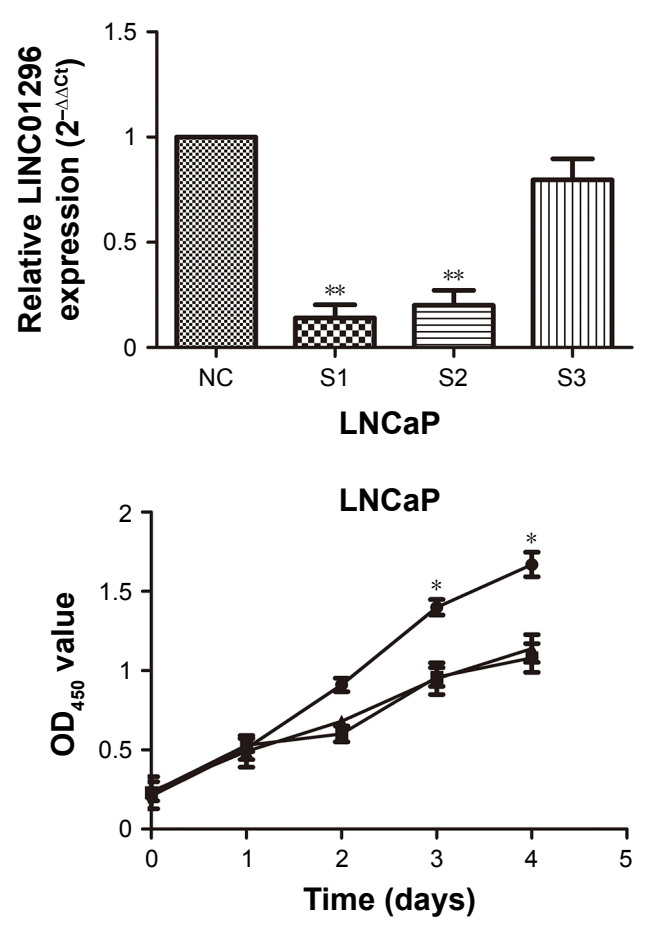

$\mathrm{NC} \rightarrow \mathrm{S} 1 \rightarrow \mathrm{S} 2$

Figure 3 (Continued) 

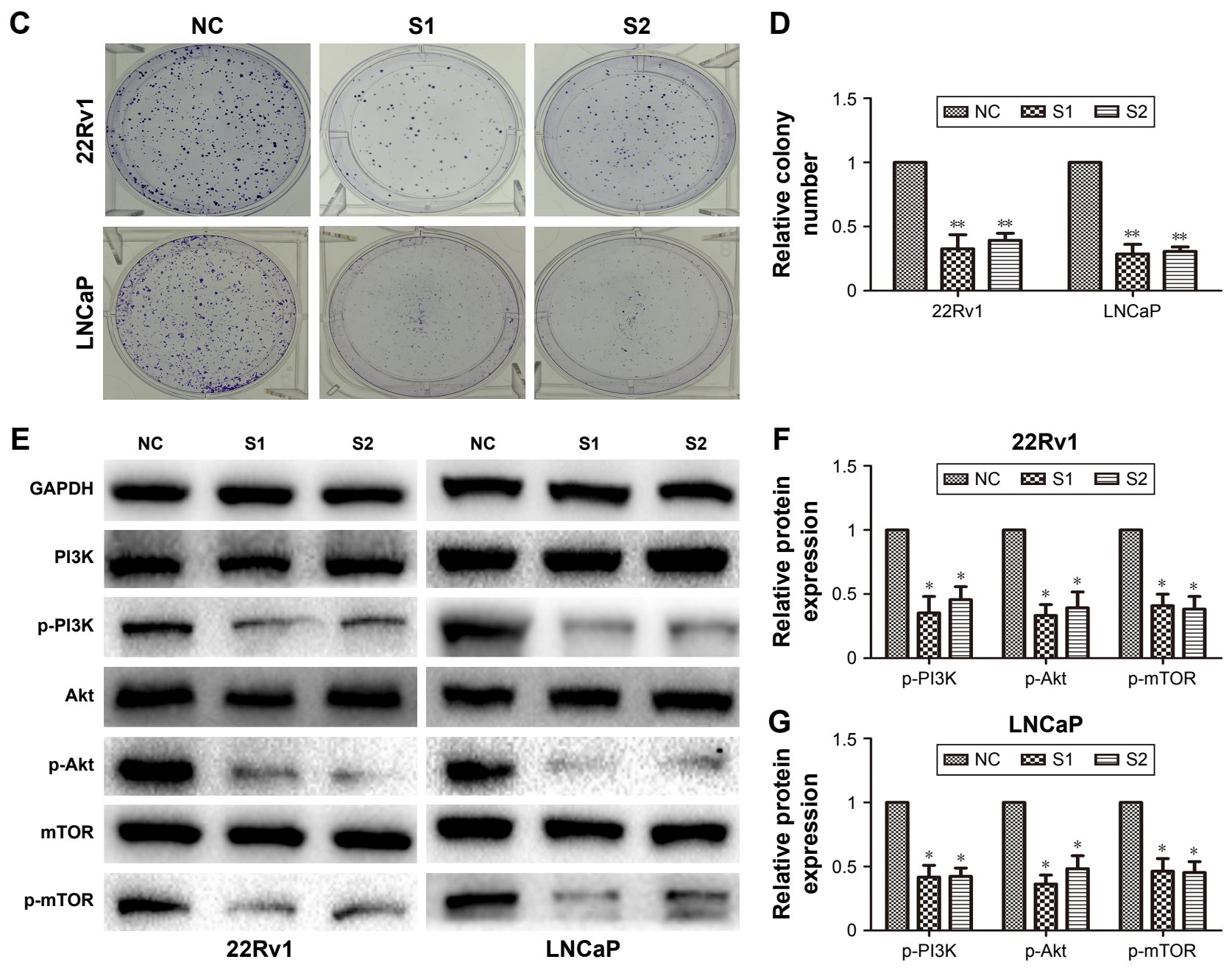

Figure 3 siRNA-mediated knockdown of LINC0I 296 inhibited prostate cancer-cell proliferation.

Notes: (A) Relative expression levels of LINC0I 296 in 22RvI and LNCaP cells were significantly decreased by siLINC0I 296 compared with the NC. (B) CCK-8 assays were performed to determine the proliferation of $22 \mathrm{RvI}$ and LNCaP cells after transfection. (C, D) Colony-formation assays were performed to determine the proliferation of siLINCO I296-transfected 22RvI and LNCaP cells. Colonies were counted and captured. (E-G) Western blot was performed to analyze relative protein-expression levels of the PI3K-Akt-mTOR signaling pathway in siLINC0I296-transfected 22RvI and LNCaP cells and respective control cells. Data represent mean \pm standard deviation from three independent experiments. $* P<0.05 ; * * P<0.01$.

Abbreviations: siRNA, short interfering RNA; NC, negative control; OD, optical density; GAPDH, glyceraldehyde 3-phosphate dehydrogenase; SI, short interfering RNAI; S2, short interfering RNA2.

Western blot analysis was used to investigate the effect of LINC01296 knockdown on the PI3K-Akt-mTOR signaling pathway. Figure 3E-G shows that LINC01296 knockdown significantly reduced the protein-expression levels of phosphorylated PI3k (p-PI3k), phosphorylated Akt (Ser473) (p-Akt), and phosphorylated mTOR (p-mTOR) $(P<0.05)$. These results suggested that LINC01296 is involved in the promotion of prostate cancer-cell proliferation and seems to be mediated by regulation of PI3K-Akt-mTOR pathway.

\section{Knockdown of LINC0I296 inhibited cell migration and invasion}

We explored the effect of LINC01296 knockdown on prostate cancer-cell migration/invasion. Figure 4A and B shows that knockdown of LINC01296 inhibited the migration capacity of $22 \mathrm{Rv} 1$ and LNCaP cells compared with cells in the NC groups $(P<0.05)$. Moreover, transwell invasion assays were performed to assess the effect of LINC01296 on the invasiveness of prostate cancer cells. The results showed that the invasion ability of siLINC01296-transfected prostate cancer cells was reduced compared with control cells (Figure $4 \mathrm{C}$ and $\mathrm{D}, P<0.05$ ). These data collectively imply that LINC01296 is involved in prostate cancer-cell migration and invasion in vitro.

Considering that LINC01296 promoted prostate cancercell migration and invasion, we further investigated the effects of LINC01296 on epithelial-mesenchymal transition (EMT) markers and $M M P 9$, a gene that is usually upregulated during 
A

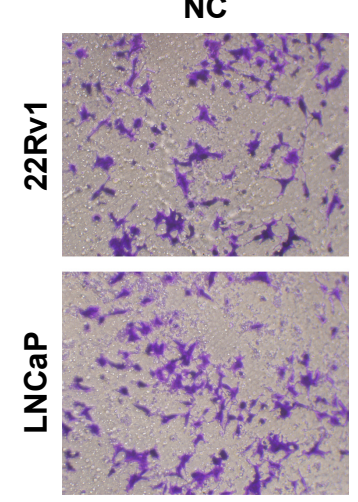

C

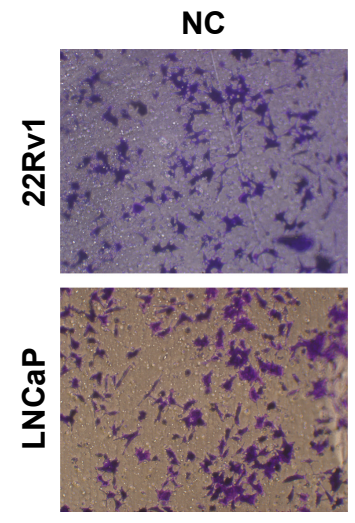

s1

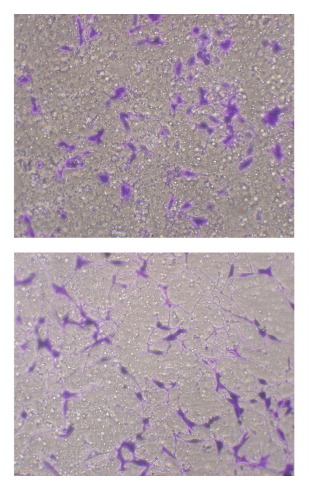

S1
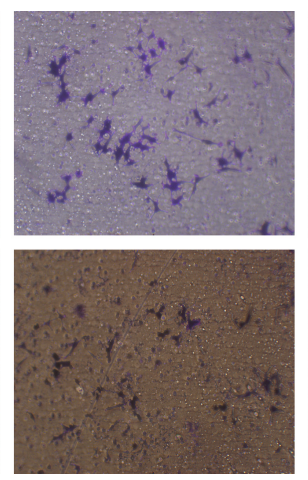

S2
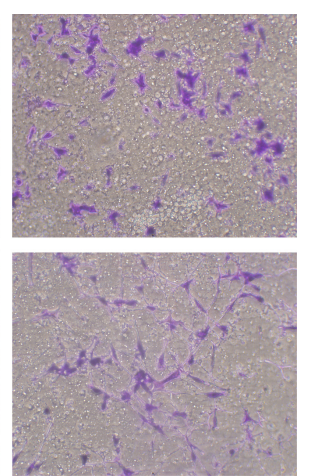

S2

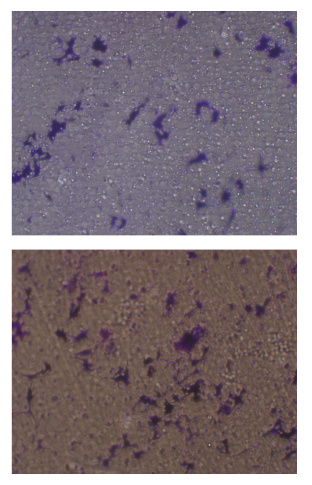

s1

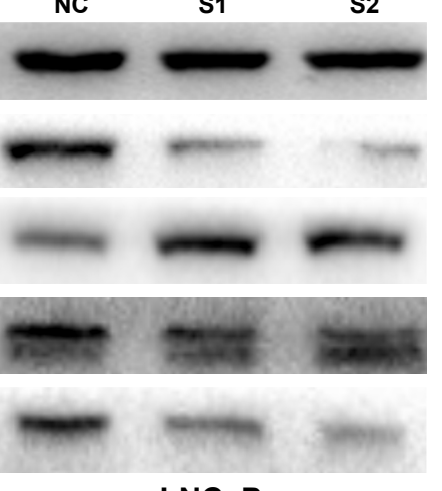

B
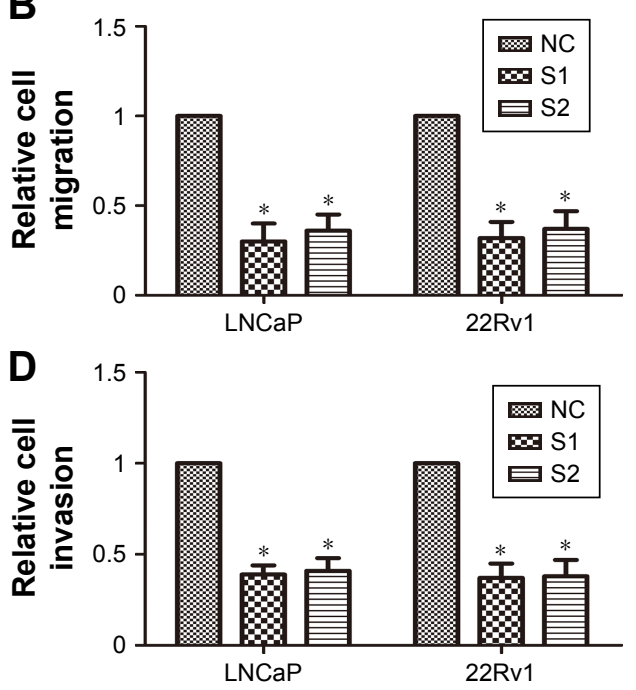

F

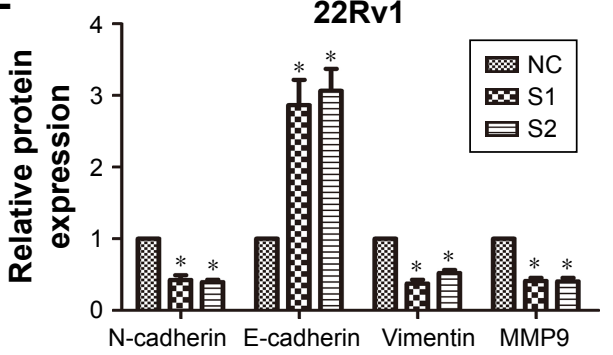

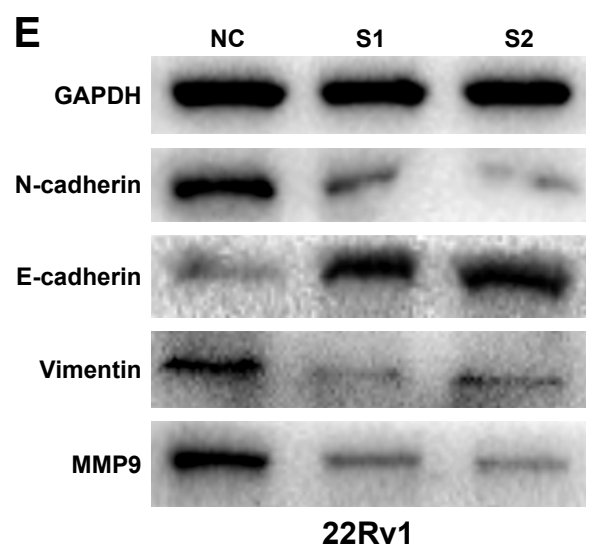

\section{LNCaP}

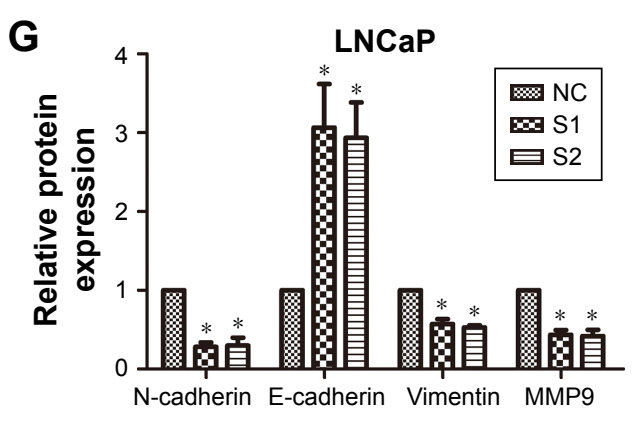

Figure 4 siRNA-mediated knockdown of LINCOI 296 inhibited prostate cancer-cell migration and invasion.

Notes: Prostate cancer cells transfected with siLINC0I 296 displayed significantly lower migration (A, B) and invasion (C, D) capacity compared with those transfected with siNC. Data represent mean \pm standard deviation from three independent experiments. (E-G) Western blot was performed to analyze of vimentin, N-catenin, E-cadherin, and MMP9 protein-expression levels in siLINC0 I296-transfected 22RvI and LNCaP cells and respective control cells. Data represent mean \pm standard deviation from three independent experiments. $* P<0.05$.

Abbreviations: siRNA, short interfering RNA; NC, negative control; GAPDH, glyceraldehyde 3-phosphate dehydrogenase; SI, short interfering RNAI; S2, short interfering RNA2.

EMT in cancer cells. ${ }^{22}$ The result of Western blot analysis demonstrated that LINC01296 knockdown increased the protein expression of E-cadherin and reduced the protein expression of N-cadherin and vimentin. Moreover, MMP9 protein expression was significantly reduced (Figure $4 \mathrm{E}-\mathrm{G}, P<0.05$ ).

\section{Discussion}

IncRNAs have recently emerged as critical elements in cell biology, as they contribute to a wide variety of cellular behaviors and functions. In cancer, lncRNAs are associated with the development and progression of tumors, contributing to essential cancer-cell functions, such as proliferation, invasion, and metastasis. ${ }^{23,24}$ Similar to other cancer types, prostate cancer is a complicated biological process characterized by myriad molecular abnormalities. IncRNAs in prostate cancer are nominated as critical actors in disease pathogenesis. ${ }^{9-19}$ Therefore, identification of cancer-associated lncRNAs and investigation of their clinical significance and functions may 
reveal the missing piece of the well-known oncogenic and tumor-suppressor network puzzle.

According to the microarray data, we future-validated and confirmed the result. In our study, we retrospectively analyzed the expression of LINC01296 in 70 prostate cancer patients and found that LINC01296 was overexpressed in prostate cancer, consistent with the microarray data. Studies have also revealed that LINC01296 is upregulated in 22Rv1 and LNCaP cell lines but not in WPMY1, which is a normal prostate myofibroblast stromal cell line. Specifically, we investigated the relationship between the expression levels of LINC01296 and the clinicopathologic features of prostate cancer patients. Our results demonstrated that prostate cancer patients with high LINC01296 expression displayed poor biochemical recurrence-free survival. More importantly, univariate and multivariate analyses demonstrated that LINC01296 should be an independent prognostic factor for prostate cancer patients, and that it plays an important role in the development and progression of prostate cancer. A previous study showed that colorectal cancer patients with high expression of LINC01296 displayed significantly worse overall survival than those with low LINC01296 expression. ${ }^{25}$ However, the biomedical functions of LINC01296 in prostate cancer remain unclear.

We performed in vitro experiments to highlight further the function of LINC01296. We focused on the identification and characterization of LINC01296 in prostate cancer and its role in proliferation, migration, and invasion. siRNAmediated knockdown of LINC01296 significantly reduced the proliferation and metastasis capability of 22Rv1 and LNCaP cells compared with the control group, suggesting that LINC01296 knockdown can suppress the development of prostate cancer. Our results suggested that high LINC01296 expression increases the malignant phenotypes of prostate cancer cells. We summarized the characteristics of LINC01296 relative to those of other previously reported lncRNAs: 1) LINC01296 expression was closely correlated with Gleason score and tumor stage of prostate cancer; 2) higher expression of LINC01296 resulted in worse biochemical recurrence-free survival; and 3) LINC01296 knockdown can significantly inhibit the growth and metastasis of prostate cancer cells. These findings suggested that LINC01296 can be used as a novel biomarker and therapeutic target in prostate cancer.

To identify the potential molecular mechanism through which LINC01296 contributes to cell proliferation of prostate cancer cells, we investigated the PI3K-Akt-mTOR signaling pathway. This is one of the three main signaling pathways, and plays an important role in the development and progression of cancer. ${ }^{26,27}$ Our previous research found that in prostate cancer, the lncRNA LOC400891 regulated cell proliferation through the PI3K-Akt-mTOR signaling pathway. ${ }^{28}$ In addition, CRNDE, an lncRNA, promotes glioma cell growth via the mTOR-signaling pathway. ${ }^{29}$ These results showed that siRNA-mediated LINC01296 silencing significantly reduced the $\mathrm{PI} 3 \mathrm{~K}-\mathrm{Akt}-\mathrm{mTOR}$ signaling pathway's relevant protein-expression level compared with NC. Therefore, we hypothesized that LINC01296 regulates the PI3K-Akt-mTOR signaling pathway via epigenetic modification, thereby promoting tumorigenesis. EMT is an important step in metastasis, during which epithelial cells are transformed into mesenchymal cells under specific physiological and pathological conditions. ${ }^{30,31}$ MMP9 is frequently observed in prostate cancer progression, ${ }^{32,33}$ and the upregulated expression of MMP9 is usually correlated with the occurrence of metastasis or local progression of cancer. ${ }^{16}$ Studies have found that lncRNA promotes cancer metastasis by regulating the EMT process. ${ }^{34,35}$ In this study, we investigated the role of LINC01296 in the EMT process in prostate cancer, and the results indicated that LINC01296 knockdown significantly suppressed the EMT process by reducing N-cadherin-, vimentin-, and MMP9-expression levels and by increasing E-cadherin expression compared with the control group. We mechanistically demonstrated that LINC01296 is possibly an inducing factor of EMT, making it an important factor in prostate cancer metastasis. lncRNAs usually bind with protein targets to perform their function in tumorigenesis. ${ }^{36}$ Therefore, the binding partner of LINC01296 should be further explored.

\section{Conclusion}

We demonstrated that increased LINC01296 expression is a common event underlying prostate cancer, suggesting that LINC01296 acts as an independent negative prognosis factor for prostate cancer patients. The effects of LINC01296 on proliferation, migration, and invasion suggest that it promotes tumorigenesis in prostate cancer. Increased LINC01296 expression promotes prostate cancer growth and metastasis by activating the PI3K-Akt-mTOR signaling pathway and EMT, and thus is a promising prognostic factor and therapeutic target to treat prostate cancer.

\section{Acknowledgment}

This work was supported by the National Science Foundation of Jiangsu Province (BK20141495).

\section{Disclosure}

The authors report no conflicts of interest in this work. 


\section{References}

1. Torre LA, Bray F, Siegel RL, Ferlay J, Lortet-Tieulent J, Jemal A. Global cancer statistics, 2012. CA Cancer J Clin. 2015;65(2):87-108.

2. Ferlay J, Soerjomataram I, Dikshit R, et al. Cancer incidence and mortality worldwide: sources, methods and major patterns in GLOBOCAN 2012. Int J Cancer. 2015;136(5):E359-E386.

3. Siegel RL, Miller KD, Jemal A. Cancer statistics, 2016. CA Cancer J Clin. 2016;66(1):7-30.

4. Ito K. Prostate cancer in Asian men. Nat Rev Urol. 2014;11(4): 197-212.

5. Chen W, Zheng R, Baade PD, et al. Cancer statistics in China, 2015. CA Cancer J Clin. 2016;66(2):115-132.

6. ENCODE Project Consortium. An integrated encyclopedia of DNA elements in the human genome. Nature. 2012;489(7414):57-74.

7. Brosnan CA, Voinnet O. The long and the short of noncoding RNAs. Curr Opin Cell Biol. 2009;21(3):416-425.

8. Zhang A, Zhang J, Kaipainen A, Lucas JM, Yang H. Long non-coding RNA: a newly deciphered "code" in prostate cancer. Cancer Lett. 2016;375(2):323-330.

9. Bussemakers MJ, van Bokhoven A, Verhaegh GW, et al. DD3: a new prostate-specific gene, highly overexpressed in prostate cancer. Cancer Res. 1999;59(23):5975-5979.

10. Salameh A, Lee AK, Cardo-Vila M, et al. PRUNE2 is a human prostate cancer suppressor regulated by the intronic long noncoding RNA PCA3. Proc Natl Acad Sci U S A. 2015;112(27):8403-8408.

11. Ren S, Liu Y, Xu W, et al. Long noncoding RNA MALAT-1 is a new potential therapeutic target for castration resistant prostate cancer. J Urol. 2013;190(6):2278-2287.

12. Prensner JR, Iyer MK, Balbin OA, et al. Transcriptome sequencing across a prostate cancer cohort identifies PCAT-1, an unannotated lincRNA implicated in disease progression. Nat Biotechnol. 2011; 29(8):742-749.

13. Prensner JR, Iyer MK, Sahu A, et al. The long noncoding RNA SChLAP1 promotes aggressive prostate cancer and antagonizes the SWI/SNF complex. Nat Genet. 2013;45(11):1392-1398.

14. Srikantan V, Zou Z, Petrovics G, et al. PCGEM1, a prostate-specific gene, is overexpressed in prostate cancer. Proc Natl Acad Sci U S A. 2000; 97(22):12216-12221.

15. Prensner JR, Sahu A, Iyer MK, et al. The IncRNAs PCGEM1 and PRNCR1 are not implicated in castration resistant prostate cancer. Oncotarget. 2014;5(6):1434-1438.

16. Parolia A, Crea F, Xue H, et al. The long non-coding RNA PCGEM1 is regulated by androgen receptor activity in vivo. Mol Cancer. 2015; $14: 46$.

17. Zhou Y, Zhang X, Klibanski A. MEG3 noncoding RNA: a tumor suppressor. J Mol Endocrinol. 2012;48(3):R45-R53.

18. Zhou Y, Zhong Y, Wang Y, et al. Activation of $\mathrm{p} 53$ by MEG3 noncoding RNA. J Biol Chem. 2007;282(34):24731-24742.

19. Zhu M, Chen Q, Liu X, et al. lncRNA H19/miR-675 axis represses prostate cancer metastasis by targeting TGFBI. FEBS J. 2014; 281(16):3766-3775.

OncoTargets and Therapy

\section{Publish your work in this journal}

OncoTargets and Therapy is an international, peer-reviewed, open access journal focusing on the pathological basis of all cancers, potential targets for therapy and treatment protocols employed to improve the management of cancer patients. The journal also focuses on the impact of management programs and new therapeutic agents and protocols on

Submit your manuscript here: http://www.dovepress.com/oncotargets-and-therapy-journal
20. Derrien T, Johnson R, Bussotti G, et al. The GenCode v7 catalog of human long noncoding RNAs: analysis of their gene structure, evolution, and expression. Genome Res. 2012;22(9):1775-1789.

21. Djebali S, Davis CA, Merkel A, et al. Landscape of transcription in human cells. Nature. 2012;489(7414):101-108.

22. Martorana AM, Zheng G, Crowe TC, O'Grady RL, Lyons JG. Epithelial cells up-regulate matrix metalloproteinases in cells within the same mammary carcinoma that have undergone an epithelial-mesenchymal transition. Cancer Res. 1998;58(21):4970-4979.

23. Esteller M. Non-coding RNAs in human disease. Nat Rev Genet. 2011; 12(12):861-874.

24. Gutschner T, Diederichs $S$. The hallmarks of cancer: a long non-coding RNA point of view. RNA Biol. 2012;9(6):703-719.

25. Qiu JJ, Yan JB. Long non-coding RNA LINC01296 is a potential prognostic biomarker in patients with colorectal cancer. Tumour Biol. 2015;36(9):7175-7183.

26. Yap TA, Garrett MD, Walton MI, Raynaud F, de Bono JS, Workman P. Targeting the PI3K-AKT-mTOR pathway: progress, pitfalls, and promises. Curr Opin Pharmacol. 2008;8(4):393-412.

27. Papadimitrakopoulou V. Development of PI $3 \mathrm{~K} / \mathrm{AKT} / \mathrm{mTOR}$ pathway inhibitors and their application in personalized therapy for non-smallcell lung cancer. $J$ Thorac Oncol. 2012;7(8):1315-1326.

28. Wang J, Cheng G, Li X, et al. Overexpression of long non-coding RNA LOC400891 promotes tumor progression and poor prognosis in prostate cancer. Tumour Biol. 2016;37(7):9603-9613.

29. Wang Y, Wang Y, Li J, Zhang Y, Yin H, Han B. CRNDE, a longnoncoding RNA, promotes glioma cell growth and invasion through mTOR signaling. Cancer Lett. 2015;367(2):122-128.

30. Kalluri R, Weinberg RA. The basics of epithelial-mesenchymal transition. J Clin Invest. 2009;119(6):1420-1428.

31. Arvelo F, Sojo F, Cotte C. Tumour progression and metastasis. Ecancermedicalscience. 2016;10:617.

32. Kessenbrock K, Plaks V, Werb Z. Matrix metalloproteinases: regulators of the tumor microenvironment. Cell. 2010;141(1):52-67.

33. Castellano G, Malaponte G, Mazzarino MC, et al. Activation of the osteopontin/matrix metalloproteinase-9 pathway correlates with prostate cancer progression. Clin Cancer Res. 2008;14(22):7470-7480.

34. Lv J, Fan HX, Zhao XP, et al. Long non-coding RNA Unigene56159 promotes epithelial-mesenchymal transition by acting as a ceRNA of miR-140-5p in hepatocellular carcinoma cells. Cancer Lett. 2016; 382(2):166-175.

35. Xu ZY, Yu QM, Du YA, et al. Knockdown of long non-coding RNA HOTAIR suppresses tumor invasion and reverses epithelial-mesenchymal transition in gastric cancer. Int J Biol Sci. 2013;9(6):587-597.

36. Prensner JR, Chen W, Iyer MK, et al. PCAT-1, a long noncoding RNA, regulates BRCA2 and controls homologous recombination in cancer. Cancer Res. 2014;74(6):1651-1660. patient perspectives such as quality of life, adherence and satisfaction The manuscript management system is completely online and includes a very quick and fair peer-review system, which is all easy to use. Visit http://www.dovepress.com/testimonials.php to read real quotes from published authors. 